\title{
DETACH: Authoring Digital Therapeutic Artefacts
}

\author{
Filipe Fernandes, Luís Duarte, Luís Carriço \\ LaSIGE \& Department of Informatics, University of Lisbon \\ Edifício C6, Campo-Grande, 1749-016, \\ Lisboa, Portugal \\ ffernandes@di.fc.ul.pt \\ lduarte@lasige.di.fc.ul.pt_1mc@di.fc.ul.pt
}

\begin{abstract}
We present DETACH, an authoring tool which allows therapists to design and deploy digital artefacts for cognitive behavioral therapy procedures. The system potentiates the creation of mobile applications by choosing screen designs from a limited template set based on existing therapeutic paper forms. Screen transitions can be customized according to patient's responses. By capitalizing on modern smart-phones' sensors and features, these transitions can also emerge from external variables such as elapsed time, current user location or user physiological state. The system's development process followed a user-centered design approach, counting with the clinicians' valuable feedback in multiple participatory design sessions. In this paper we present the early design stages, clinicians' requirements, and how these translated into both our low-fidelity and high-fidelity prototypes. Usability evaluation is still undergoing, with multiple test sessions taking place to guarantee the tool fully adheres to the stakeholders' expectations.
\end{abstract}

Keywords-Authoring Tool; Cognitive Behavioral Therapy; Participatory Design

\section{Motivation}

Clinical applications for mobile phones have been successfully used to improve a patient's state [3][7]. The increasing dissemination and power of smart-phones has further emphasized this possibility [2][7]. They provide: clinicians with sophisticated means to more closely accompany their patients in their daily lives; patients with proactive and easy to use tools addressing their issues. Success cases for pathologies and therapy procedures as diverse as autism [1] or fear therapy [2] are testaments on the benefits of technology.

Nevertheless, a significant number of these applications fall short to success. Several factors can account for this outcome, among which the inability to personalize and adapt content [8]. For instance, an application's presentation is typically the same for all users who download it. Yet, the expectations of a potential 8 year old user are quite different from those of a 45 year old patient. Also, the evolution of the patient's health status often requires adjustments that applications are not ready to accompany. For example, monitoring thresholds vary and support messaging and data collection should be adapted to new clinical assessments.

The origin of this application stiffness builds in many factors. The complexity of the technology and of the application domain is certainly one of those reasons. In fact we believe that it is one of the most important factors: the dichotomy and complexity of knowledge involved. IT engineers and researchers dominate the technology and are in general able to handle its complexity. Clinicians on the other end understand patients and the protocols they must put forward to provide them a better quality of life. Combining the two knowledge sources is no easy task. It gets worst because both knowledge domains evolve rapidly as well as the ultimate target, the patient wellbeing status.

The usage of authoring tools is a possible solution. Past works prove valuable evidence in tackling similar situations [1]. These tools aim at joining two knowledge sources to a middle ground. They provide domain experts the mechanisms to customize and deeply adapt the applications, refining and embedding it with clinical decisions. For that, the tools must hide the technology complexity under a hopefully well-defined set of components, developed by IT staff. At the end they offer means for end-user programming. Finding that adequate middle-ground can be complicated. It is not just about usability. It is also about programming and domain concepts, and ultimately the domain experts' perception of its combination

In this paper we propose DETACH - DEsign Tool for smartphone Application Composition in the Health domain - a tool that allows therapists to compose ubiquitous applications. It provides a set of building customizable blocks that can be connected according to the patients' explicit or implicit behavior. Domain experts are the tool's end-users and thus responsible to define the applications according to the adequate clinical procedures and evolution.

\section{BACKGROUND}

Our research is framed within the domain of Cognitive Behavioral Therapy (CBT). This is a form of psychotherapy that emphasizes on what we do and how we feel, focusing the patients' current problems [9]. Therapists often request patients to keep a diary in which they store daily emotions, thoughts and feelings towards specific situations. Traditionally, this is done recurring to a paper questionnaire and filling it in at appropriately. State-of-the-art research shows that replacing paper with digital artefacts is a well embraced approach [7]. 


\section{DESIGN PROCESS}

A participatory design process was chosen as the approach to create this authoring environment (Fig. 1). We have conducted a substantial set of sessions with 15 clinicians to understand: a) current clinicians' requirements and expectations for a tool of this nature; b) how modern smartphones can improve existing therapeutic practices without disrupting standing practices; c) interactions patterns employed by our stakeholders when designing and organizing different elements of an application.

\section{A. Requirements}

The design sessions encompassed a team of expert HCI researchers along clinicians specializing in different types of interventions and for different pathologies. We assessed what would be the main expectations for them to operate a system of this nature. Using the thoughtful interaction design methodology, we were able to identify the following requirements: a) existence of a broad set of template designs with editable elements (e.g. text questions/answers, images); b) configurable screen transitions based, among other, on patient answers and / or screen sequential order; c) functionality which would enable clinicians to preview the application according to what the patients would experience (e.g. an emulator).
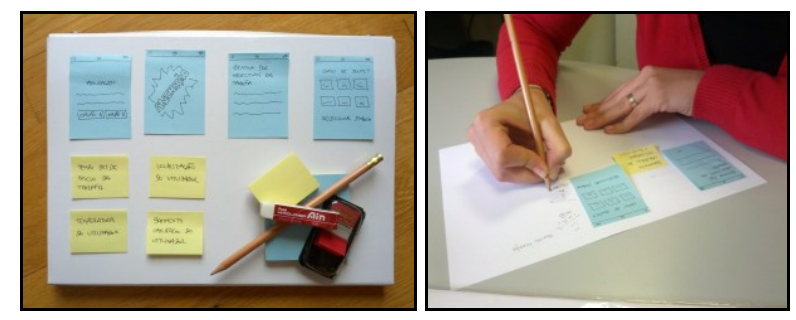

Fig. 1 - Participatory design sessions: used material (left); low-fidelity prototype testing (right).

\section{B. Capitalizing on Modern Smart-Phones}

After identifying the DETACH's main requirements, we carried a couple of sessions in which we introduced the therapists to the features of modern smart-phones (e.g. Android 2.1 phones or above), asking them how they felt patients would benefit from them. Clinicians were given freedom to envision scenarios using the smart-phone's features and were required to express it using our low fidelity prototype setup (Fig.1). The most common scenarios encompassed: a) tracking the patient's location / time of day to trigger the device to warn him / her about filling-in a digital form when in the vicinity of certain locations; b) physiological state tracking to prompt relaxing animations, music or other type of media, when the application detects values above certain thresholds for that patient.

\section{High-Fidelity Prototype}

Based on stakeholder feedback, we designed a high-fidelity prototype (Fig. 2). The top section displays the available screen templates (e.g. multiple choice answer, animation display, etc.). Clinicians can drag a template into the center canvas and configure each screen's particular elements on the rightmost panel. Transitions between screens (represented by arrows) are defined by selecting a screen, hitting a button on top of it and finally stipulate the rules which trigger the transition.

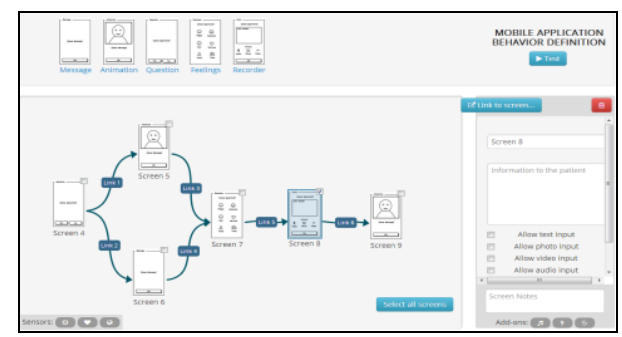

Fig. 2 - Authoring tool high-fidelity prototype.

In the lower area of the canvas, the clinician can toggle the usage of smart-phone sensors and features. Consequently, richer transition rules can be defined: if the patient is near a determined location, then the application would trigger an alarm to warn him / her to fill-in the digital form; if the patient's average heartbeat rate is above a specified threshold, then the application would play a relaxing tune or a motivational animation.

\section{FUTURE WORK}

We are currently working on the digital artefact previewer, XML exporting for the artefacts and testing each feature with our stakeholders. The final step of this research will encompass a set of clinical trials in which we will assess whether authored applications can foster patient commitment when compared to previous non-authored digital artefacts.

\section{ACKNOWLEDGEMENTS}

This work was funded by FCT, through Individual Scholarship SFRH / BD / 39496 / 2007, through project PTDC/EIA-EIA/117058/2010 and the Multiannual Funding Programme.

\section{REFERENCES}

[1] Boujarwah, F. A., et al. 2011.REACT: intelligent authoring of social skills instructional modules for adolescents with high-functioning Autism. SIGACCESS Access. Comput. 99 (January 2011), 13-23.

[2] Carriço, L., Sá, M., Duarte, L., Reis, T. 2008. A Mixed-Fidelity Prototyping Tool for Mobile Devices. In Procs of AVI '08., 225-232.

[3] Das, A. K., 2002. Computers in Psychiatry: A Review of Past Programs and an Analysis of Historical Trends. In Psychiatry Quarterly, No.79 (4).

[4] Hartmann, B., et al. d.tools: Visually Prototyping Physical UIs through Statecharts. In Ext. Abstracts of ACM Symposium on User Interface Software and Technology (UIST 2005), 2005.

[5] H.Lieberman, F.Paternó, W.Wulf (eds), End-User Development, Springer Verlag, ISBN-10 1-4020-4220-5, 2006.

[6] Lowgren, J. et al. Thoughful Interaction Design. MIT Press, 2004.

[7] Matthews, M., Doherty, G. 2011. In the mood: engaging teenagers in psychotherapy using mobile phones. In Procs of CHI '11. 2947-2956.

[8] Offer, D., K.I. Howard, K.A. Schonert, and E. Ostrov, "To whom do adolescents turn for help? Differences between disturbed and nondisturbed adolescents." Journal of the American Academy of Child and Adolescent Psychiatry 1991.30(4) p. pp. 623-630.

[9] Timms, P. et al. "Cognitive Behavioral Therapy", In leaflet for the Royal College of Psychiatrists' Public Education Editorial Board, March, 2007 